\title{
AMENDMENTS
}

\section{Publisher Correction: Resonant phase-matching between a light wave and a free-electron wavefunction}

Raphael Dahan, Saar Nehemia, Michael Shentcis, Ori Reinhardt, Yuval Adiv, Xihang Shi, Orr Be'er, Morgan H. Lynch, Yaniv Kurman, Kangpeng Wang and Ido Kaminer (iD

Correction to: Nature Physics https://doi.org/10.1038/s41567-020-01042-w, published online 12 October 2020.

In the version of this Article originally published, the received date of 21 April 2020 was incorrect and should have been 15 October 2019. This has now been corrected in the online versions of the Article.

Published online: 22 January 2021

https://doi.org/10.1038/s41567-021-01178-3

(๑) The Author(s), under exclusive licence to Springer Nature Limited 2021

\section{Publisher Correction: Enhanced thermal Hall effect in the square-lattice Néel state}

Rhine Samajdar (D), Mathias S. Scheurer (D), Shubhayu Chatterjee (D), Haoyu Guo (D), Cenke Xu (D) and Subir Sachdev (D)

Correction to: Nature Physics https://doi.org/10.1038/s41567-019-0669-3, published online 7 October 2019.

In the version of this Article originally published, in ref. 3, the journal name 'Phys. Rev.' was incorrect and should have been 'Phys. Rev. B'. The online versions of the Article have been amended.

Published online: 26 January 2021

https://doi.org/10.1038/s41567-021-01180-9

(c) The Author(s), under exclusive licence to Springer Nature Limited 2021

\section{Publisher Correction: Bacteria solve the problem of crowding by moving slowly}

\author{
O. J. Meacock (D), A. Doostmohammadi (D), K. R. Foster (D), J. M. Yeomans (D) and W. M. Durham (D)
}

Correction to: Nature Physics https://doi.org/10.1038/s41567-020-01070-6, published online 23 November 2020.

In the version of this Letter originally published online, the author J. M. Yeomans was incorrectly affiliated with 'Niels Bohr Institute, University of Copenhagen, Copenhagen, Denmark', instead of 'Rudolf Peierls Centre for Theoretical Physics, Clarendon Laboratory, University of Oxford, Oxford, UK'. This affiliation has now been added, and other footnotes renumbered accordingly, in all versions of the Letter.

Published online: 28 January 2021

https://doi.org/10.1038/s41567-021-01181-8

(C) The Author(s), under exclusive licence to Springer Nature Limited 2021 